\title{
Wave packet structure and phase jumps in low latitude Pc3 geomagnetic pulsation
}

\author{
I A ANSARI, B J FRASER* and K J MCLEAN \\ Department of Electrical and Computer Engincering, University of Wollongong, NSw 2500, \\ Australia \\ *Department of Physics, University of Newcastle, NSW 2308, Australia \\ MS received 7 December 1984; revised 17 October 1985 \\ Abstract. An array of four low latitude induction coil magnetometer stations has been used \\ to study the spatial and temporal characteristics of $\mathrm{Pc} 3$ pulsations over a longitudinal range of \\ $17^{\circ}$ at $L=1.8$ to 2.7 in south-east Australia. A preliminary study of individual $\mathrm{Pc} 3$ wave \\ packet structure at the azimuthal stations has established the existence of phase jumps \\ between wave packets at low latitudes similar to those observed at synchronous orbit and at \\ higher latitude ground stations. However there did not appear to be any obvious pattern in \\ phase jump occurrences between stations or signal components.
}

Keywords. Pc3 geomagnetic pulsations; wave packet; phase jump; magnetosphere; field line resonance.

\section{Introduction}

Pc3 geomagnetic pulsations are quasisinusoidal variations in the earth's magnetic field in the period range 10 to $45 \mathrm{sec}$ (Jacobs 1970). These pulsations are characterized by wave-train envelopes which show a regular beat-like modulation. Herron (1966) investigated the phase characteristics of Pc3-4 signal wave packets at mid-latitudes and observed discrete phase changes in the pulsation signal when the amplitude of the signal between wave packets became small. In their study of longitudinal phase variations in high latitude Pc4 pulsations Olson and Rostoker (1978) observed sudden phase changes but did not study them in detail. Using data from three synchronous satellites and ground data, Mier-Jedrzejowicz and Hughes (1980) have shown that the phase jumps occur between wave packets in dayside pulsation signals. Signals in a single wave packet from two stations may drift apart in phase and are brought back into phase as a result of phase jumps. They suggested that the changes in Pc3 wave polarization are due to phase skips in either the $H$ or the $D$ component. They further found that these phase skips seldom occur simultaneously at all latitudes.

Phase skipping is an important property in Pc3-5 pulsations. It may provide an insight into the impulsive, temporal or spatial variations associated with the wave generation mechanism.

\section{Data and analysis}

An array of four low latitude induction coil magnetometer stations was established in south-east Australia to accomplish the study. The array is shown in figure 1 and station 
locations and interstation distances are listed in tables $1 \mathrm{a}$ and $\mathrm{t}$ respectively. A geomagnetic longitude range of $17^{\circ}$ was covered at $L=1.8^{+}$and a latitudinal range of $10^{\circ}$ over $L=1.8$ to $2 \cdot 7$.

The block diagram of the recording system is shown in figure 2. Fluctuations in the geomagnetic field induced a small voltage in the detector coil which was then amplified

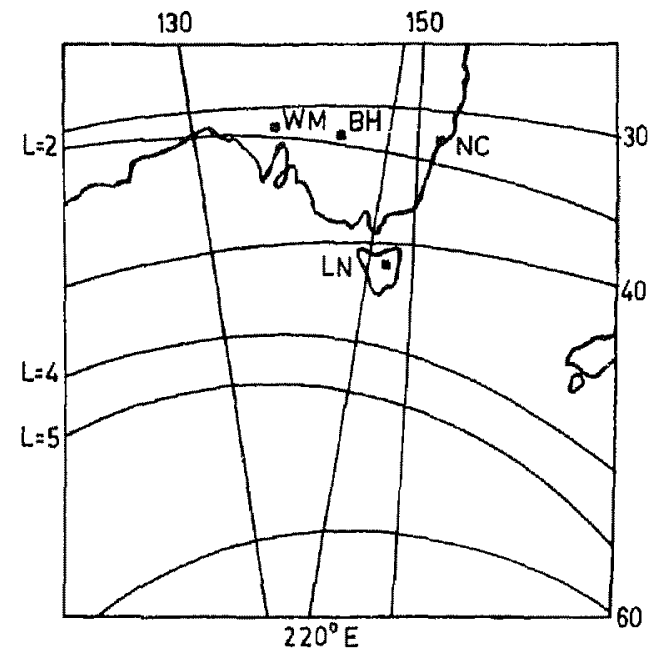

Figure 1. The University of Newcastle Pc3 recording station network.

Table 1a. Coordinate details of four Pc3 recording stations in Australia

\begin{tabular}{lcccccc}
\hline & \multicolumn{2}{c}{ Geographic } & & \multicolumn{2}{c}{ Geomagnetic } & \\
\cline { 2 - 3 } \multicolumn{1}{c}{ Stations } & $\varphi\left({ }^{\circ} \mathrm{S}\right)$ & $\lambda\left({ }^{\circ} \mathrm{E}\right)$ & & $\varphi\left({ }^{\circ} \mathrm{S}\right)$ & $\lambda\left({ }^{\circ} \mathrm{E}\right)$ & $L$ valuc \\
\hline Woomera (WM) & 31.1 & 136.7 & & $41 \cdot 7$ & 209.1 & 1.79 \\
Broken Hill (BH) & 32.0 & 141.5 & & 42.4 & 214.5 & 1.81 \\
Newcastle (NC) & 32.6 & 151.7 & & 42.0 & 226.3 & 1.81 \\
Launceston (LN) & 41.7 & 147.2 & & 52.4 & 231.1 & 2.69 \\
\hline
\end{tabular}

Table 1b. Interstation distances of the four Pc3 recording stations in Australia

\begin{tabular}{lc}
\hline Station pairs & Interstation distances $(\mathrm{km})$ \\
\hline WM-BH & 425 \\
BH-NC & 1065 \\
WM-NC & 1485 \\
NC-LN & 1120 \\
\hline
\end{tabular}

\footnotetext{
+ For a pure dipole field the parameter $L$ is the maximum radial distance of a line of force from the dipole and is measured in earth radii. The two ends of the line of force determine the magnetic latitudes e.g. $L=4$ for a magnetic latitude of $60^{\circ}$ approximately.
} 


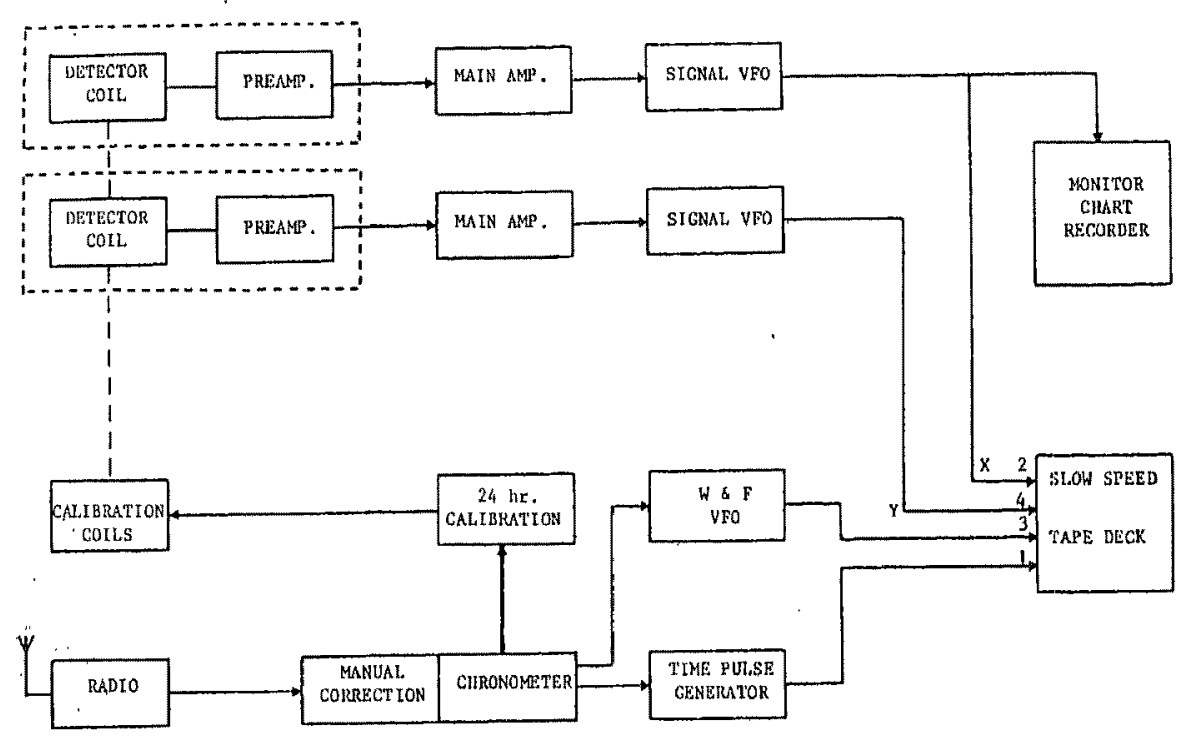

Figure 2. Block diagram of $\mathrm{Pc} 3$ recording system.

by a solid-state preamplifier situated within the coil containers and a main amplifier $100 \mathrm{~m}$ away in the equipment caravan. A frequency modulated recording system with a variable frequency oscillator ( $\mathrm{VFO}$ ) centre frequency of $6.25 \mathrm{~Hz}$ was employed to record the geomagnetic north-south $(X)$ and east-west $(Y)$ components of $\mathrm{Pc} 3-4$ wave signals in the $5-100 \mathrm{mHz}$ (milli Hertz) band using slow speed magnetic tapes. In addition to the two signal VFo, a $6.25 \mathrm{~Hz}$ square wave signal was recorded to provide wow and flutter correction during analysis. The minimum detectable signal was $0.1 \mathrm{nT}$ at $50 \mathrm{mHz}$. The timing system consisted of a chronometer accurate to 10 millisec day ${ }^{-1}$ which provided regular $5 \mathrm{sec}$ pulses with 1 and $20 \mathrm{~min}$ interval pulses delayed by 1.5 and $3.5 \mathrm{sec}$ respectively. At 24-hour intervals a range of four frequencies from 25 to $60 \mathrm{mHz}$ were fed to the detector coil to provide frequency calibration over the operating bandwidth of the system. The slow speed tape deck operated with a transport speed of approximately $57 \mathrm{~cm} \mathrm{hr}^{-1}$ and had four recording channels on a $1 / 4$ inch tape. These were the $5 \mathrm{sec}$ time pulses, the $X$ channel, the wow and flutter signal and the $Y$ channel respectively. An Easterline Angus chart recorder operating at $3.8 \mathrm{~cm} \mathrm{hr}^{-1}$ provided a visual monitor of the $X$ channel.

Both analog and digital analysis procedures were applied to the recorded data (figure 3). Data from 25 March to 21 September 1982 were digitized in the laboratory with a $5 \mathrm{sec}$ sample rate using the recorded time channel pulses and providing a Nyquist frequency of $100 \mathrm{mHz}$ (Ansari 1984). The resulting $X(t)$ and $Y(t)$ time series (see for example, figure 4) for each station were used to construct digital sonagrams over one day ( $25-\mathrm{hr}$ ) intervals using the maximum entropy method (MEM) on $10 \mathrm{~min}$ subsets overlapping by 5 min.

In order to obtain a simpler and averaged visual representation of frequency-time structure, contours at four different power spectral levels were plotted. A program CONTPT (see appendix) which uses the FFT method of calculating the power spectrum and employs the interactive graphics library (IGL) plotting routines, was used. The plots were normally obtained on a Tektronic- 4025 graphics terminal and a 4612 hard 


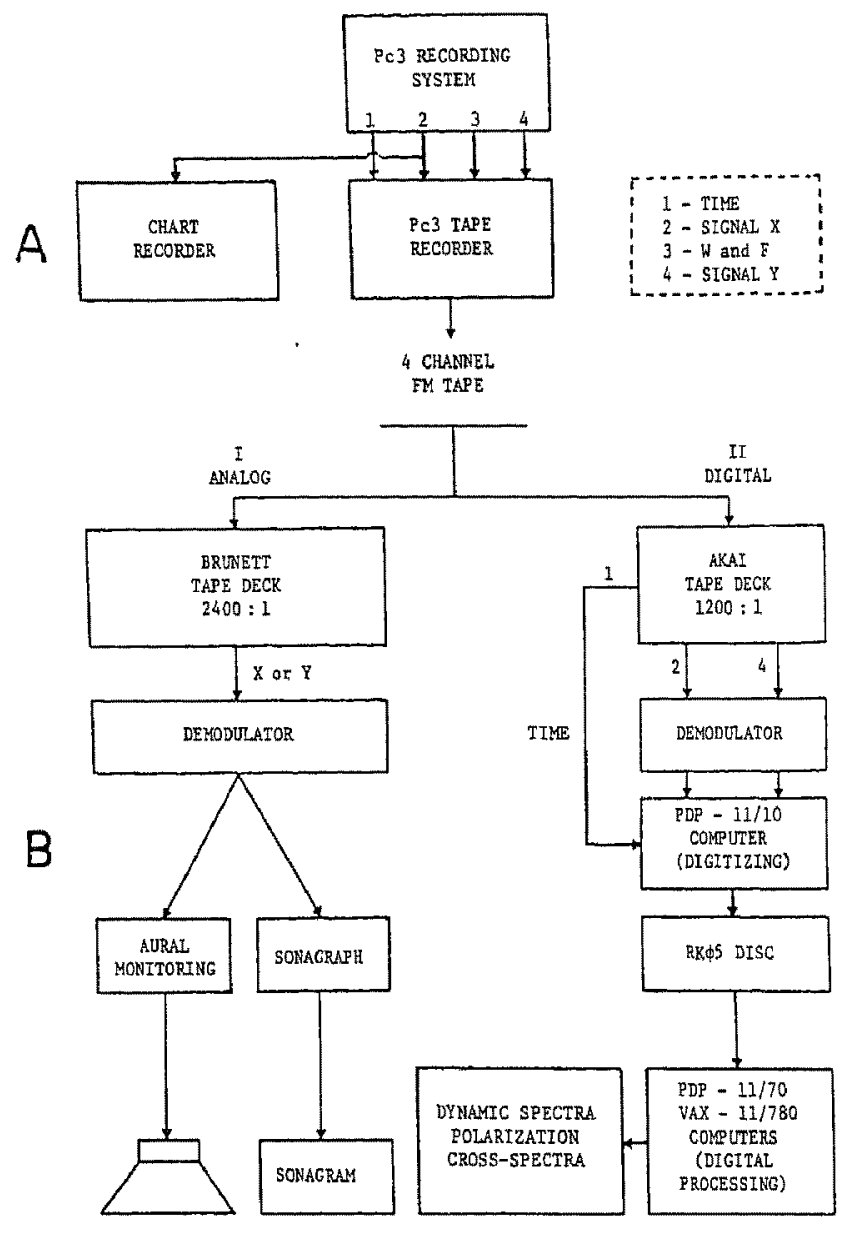

Figure 3. Pc3 data processing sequences. (A) Field recording instrumentation. (B) Laboratory analysis instrumentation.

copy unit or, were constructed on the plotter at the University of Newcastle computing centre. Plots were obtained for the duration of the Pc3 activity over four- and eighthour time intervals. An example of contour sonagrams representing four power contour levels on May 30,1982 at all four stations WM, BH, NC and LN is shown in figure 5. From these plots more precise time durations of $\mathrm{Pc} 3$ activity were measured to the nearest $10 \mathrm{~min}$. These segments are indicated by horizontal lines on the time axis. Time is always stated in local time which is conveniently close to the Australian eastern standard time $($ AEST $=\mathrm{UT}+10 \mathrm{hr})$.

\section{Results}

The properties of individual $\mathrm{Pc} 3$ wave packet structures are important since these may relate to the phase properties of the wave generation mechanisms. Wave packet 


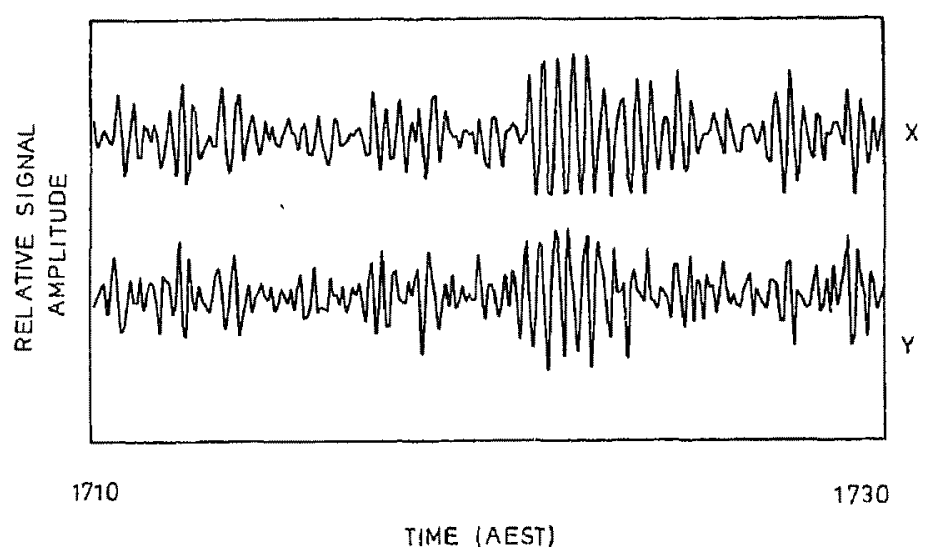

Figure 4. An example of an amplitude time record of a series of $P c 3$ wave trains recorded at Broken Hill on September 20, 1982 between the time intervals of 1710 to $1730 \mathrm{hr}$ AEST.

structure and phase jumps were studied for March 25, 26, 27, 28, 30 and May 27, 30, 1982 in all selected time intervals at individual and pairs of stations for both $X$ and $Y$ components. The study was also carried out for wM data of August 31, 1982. The programs WPS and PSHFT (see appendix) were developed to carry out the analysis. The results of a few examples are considered here.

Figure 6 shows the variations in the $X$ component at wM between $1410-1430 \mathrm{hr}$ AEST on August 31, 1982. Packet structure can be seen in the upper plot and clearly identifiable wave packets are labelled sequentially. In the lower plot the vertical axis is the wave cycle number counts and the times of the peaks are plotted horizontally. A constant phase difference between cycles will appear as a straight line segment in the plot. A discontinuity will indicate a sudden phase change while a change in gradient will indicate a change in frequency. It can be seen that the five wave packets have constant phase over their duration and their frequencies are the same as indicated by similar slopes. Phase jumps can be seen clearly at B, D, F and $G$ in figure 6 .

It is of interest to compare the cycle-by-cycle signal phase difference between stations for individual wave packets. Figure 7a shows the interstation phase comparison for WM and BH $X$-components over four wave packets. Here the time difference between the corresponding signal peaks is plotted on the vertical axis in the bottom graph. A horizontal line of plotted points indicates constant phase between the two stations while vertical points indicate variable phase. A sudden discontinuity relates to a phase jump. The absolute value of the time differences have little meaning since they depend on the relative alignment of the nearest corresponding peaks from the two stations. In figure $7 \mathrm{a}$ four wave packets are identified. The phase difference for packet 1 increases gradually and then drops to zero at B. Constant phase is seen between B and C over wave packet 2. Packet 3 shows a variable phase difference similar to packet 1 while the short wave packet 4 is constant in phase difference. Phase jumps are seen at $C$ and D.

Figure $7 \mathrm{~b}$ shows the phase differences over the same data interval and wave packets for BH and NC $X$-components. Variable phase differences are seen for packets 1 and 2 

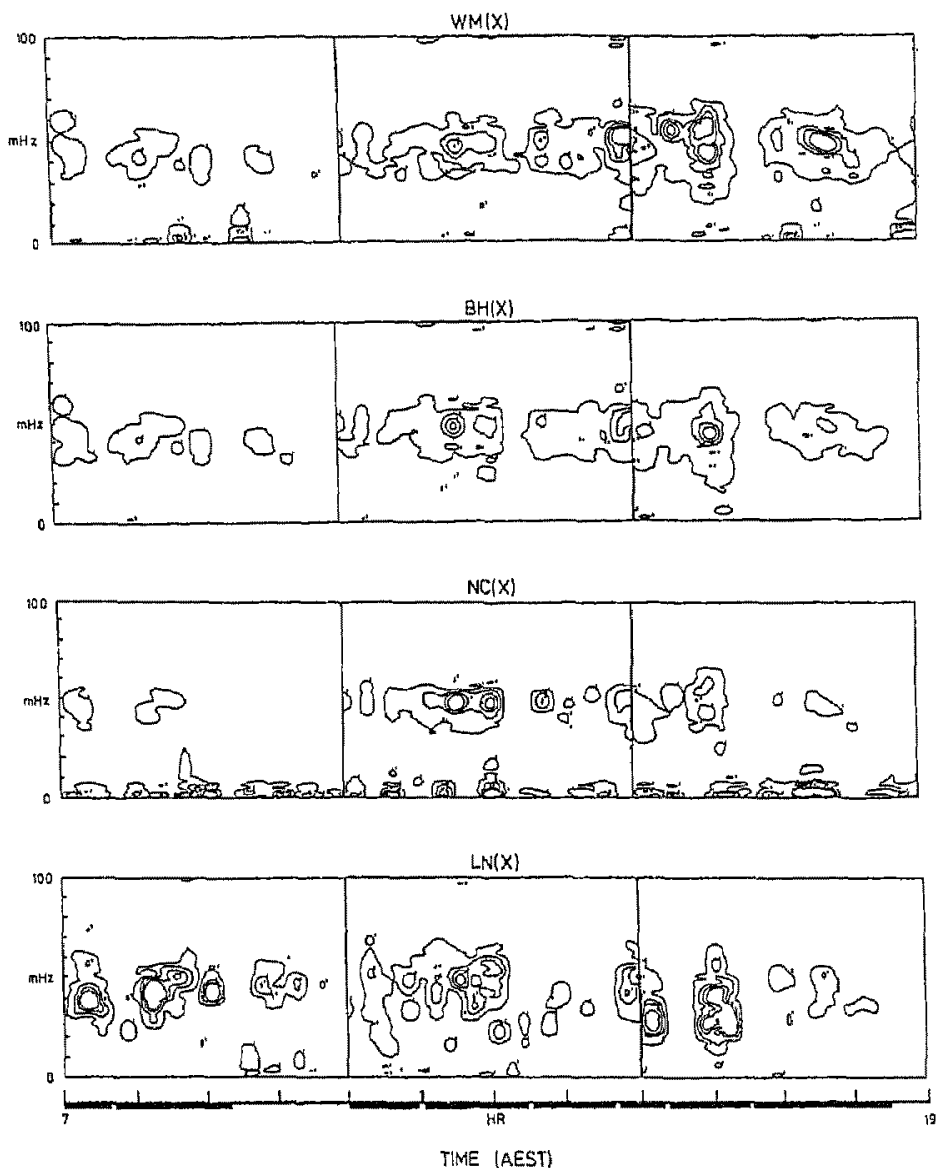

Figure 5. Contour sonagram showing Pc 3 activity on the $X$ component at the four stations WM, BH, NC and LN on May 30, 1982 between $0700-1900$ hr AEST. The segments marked with a horizontal line on the time axis show continuous pulsation activity.

with an apparent periodicity of about $80 \mathrm{sec}$ while packet 3 is constant. Packet 4 has a low amplitude at $\mathrm{NC}$ and the measurements are probably unreliable. Here phase jumps are seen at $C$ and possibly $D$ in agreement with the wM-BH station pair.

Figures $8 \mathrm{a}$ and $\mathrm{b}$ show $X$ and $Y$ phase difference data for the WM-BH and BH-NC azimuthal pairs of stations at $1210-1220 \mathrm{hr}$ AEST on May 30, 1982. Considering figure $8 \mathrm{~b}$ which shows a simpler pattern on the $Y$ component, it can be seen that the phase difference is reasonably constant over packets 1,2 and possibly 5 at WM-BH and 1 and 4 at $\mathrm{BH}-\mathrm{NC}$. The other packets show some variability. Phase jumps occur at $\mathrm{WM}-\mathrm{BH}$ 


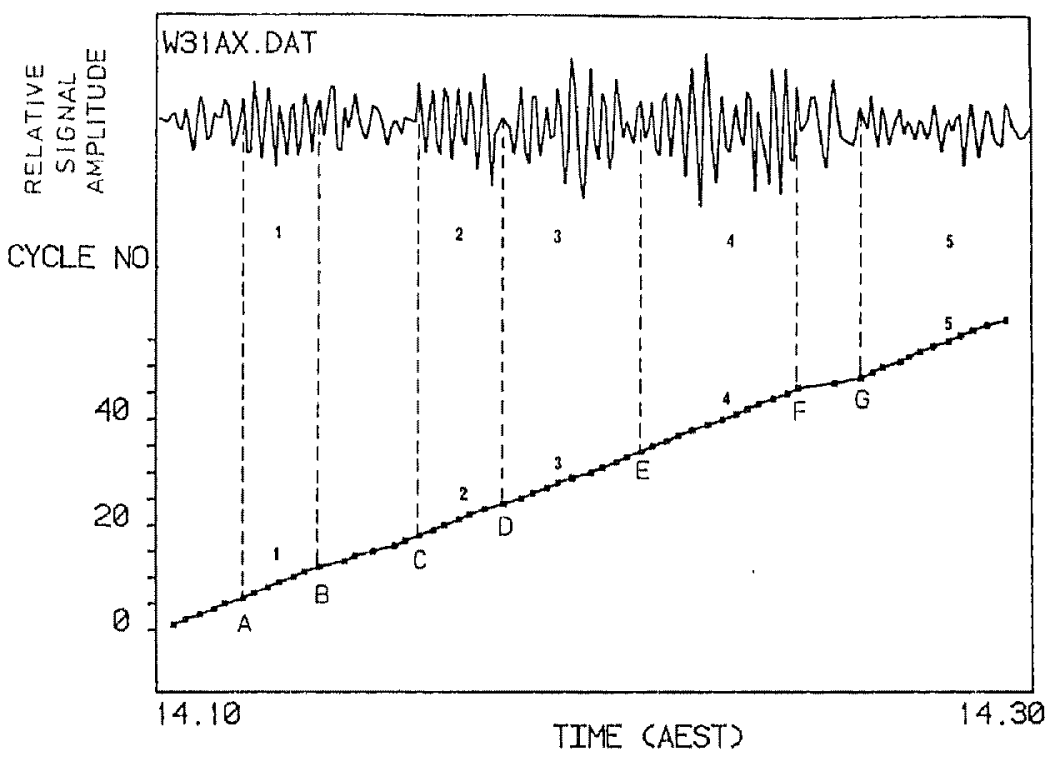

Figure 6. The amplitude-time data at the top of the figure indicates the wave packet structure in the WM $X$ component on August 31,1982 (W31AX.DAT) between $1410-1430 \mathrm{hr}$ AEST. The plotted dots at the bottom of the figure correspond to amplitude peaks in the magnetic field data.

at $\mathrm{C}$ and at different times at $\mathrm{BH}-\mathrm{NC}$ namely at $\mathrm{B}$ and $\mathrm{E}$. The $X$ components show a different packet structure with the $N C$ data, the boundaries $D$ and $E$ shifted to $D^{\prime}$ and $E^{\prime}$ respectively. Phase differences are reasonably constant over packets 2 and 4. Data are variable in all packet time intervals at the WM-BH pair. Phase jumps occur near $D^{\prime}$ and possibly $\mathrm{B}$ at both station pairs but at $\mathrm{F}$ for only the $\mathrm{BH}-\mathrm{NC}$ pair.

This example shows that there is more similarity between longitudinal $X$ components at spaced stations and also the $Y$ components than between the $X$ and $Y$ components at a single station. Furthermore, no consistent pattern of phase jumps is seen between components or station pairs.

Another example of interstation wave packet phase structure is shown in figure 9 for the afternoon interval $1800-1820 \mathrm{hr}$ AEST. Wave packets 1 to 6 were identified using the WM and вH $X$-component data as reference, since these showed the clearest packet structure. For the WM-BH station pair constant phase difference on the $X$ components is seen over packets $1,2,4$ and 6 while for the BH-NC pair constant phase is seen only in packet 2 . On the $Y$ components packets 1 and 2 show reasonably constant phase differences at both pairs of stations. Variable phase is seen over all other packets at both pairs of stations. It should be noted that the wave amplitude is reasonably low on the NCY component and this may contribute to some of the variability seen in the BHNC data for this component. The largest phase jump is seen between packets 2 and 3 at $\mathrm{B}, \mathrm{C}$ but only on the $Y$ component at the WM-BH stations and the $X$ component at the BH-NC stations. The only other significant jump is at $\mathrm{E}$ for the WM-BH stations $X$ component signal but it is not accompanied by a corresponding jump on the $Y$ component or seen at the other station pair. 


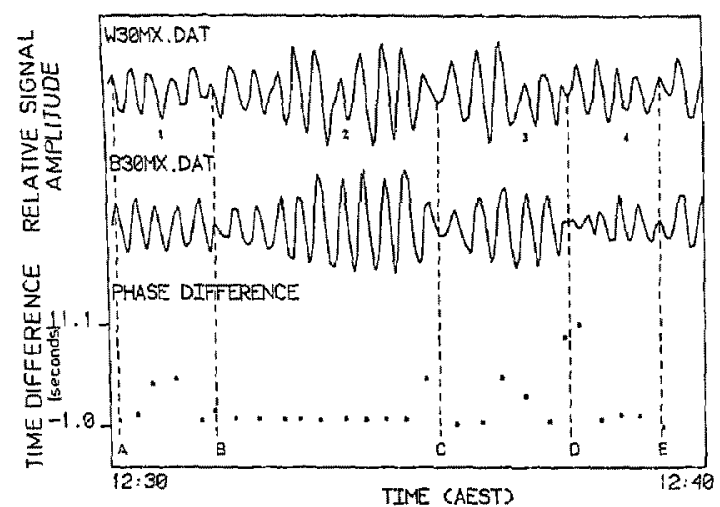

(a)

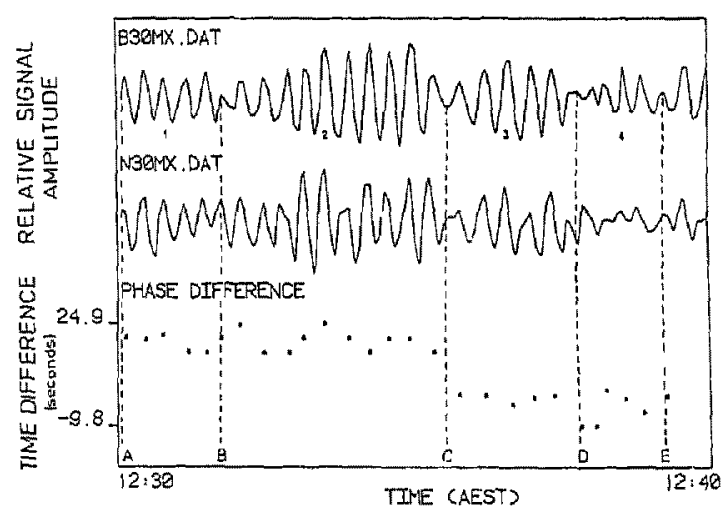

(b)

Figure 7. Interstation wave packet phase structure for May 30,1982 between $1230-1240 \mathrm{hr}$ AEST. The two $X$ wave forms show four wave packets at (a) WM and BH, (b) BH and NC. The bottom plot is the time difference between nearest corresponding peaks at the two stations. The time difference is positive if the station at the top of the panel leads in phase and negative if it lags.

\section{Discussion and conclusion}

These results on dividual $\mathrm{Pc} 3$ wave packet structure show that phase jumps are observed at low latitudes but the data do not show any systematic temporal or spatial patterns. More cases of constant phase within individual wave packets are seen for the WM-BH station pair. It may be significant that these stations are spaced only half the distance apart compared to the BH-NC stations.

With field line resonance when the driving force in the magnetosphere is removed each resonant shell will oscillate at its own resonant frequency as the signal decays (Southwood 1975). Mier-Jedrzejowicz and Hughes (1980) while explaining their observed phase shifts have proposed that some impulsive or short lived event may initiate the wave packets in phase at all stations and the excited local field lines may act as resonators. Because of slight variations in particle population or magnetic field 

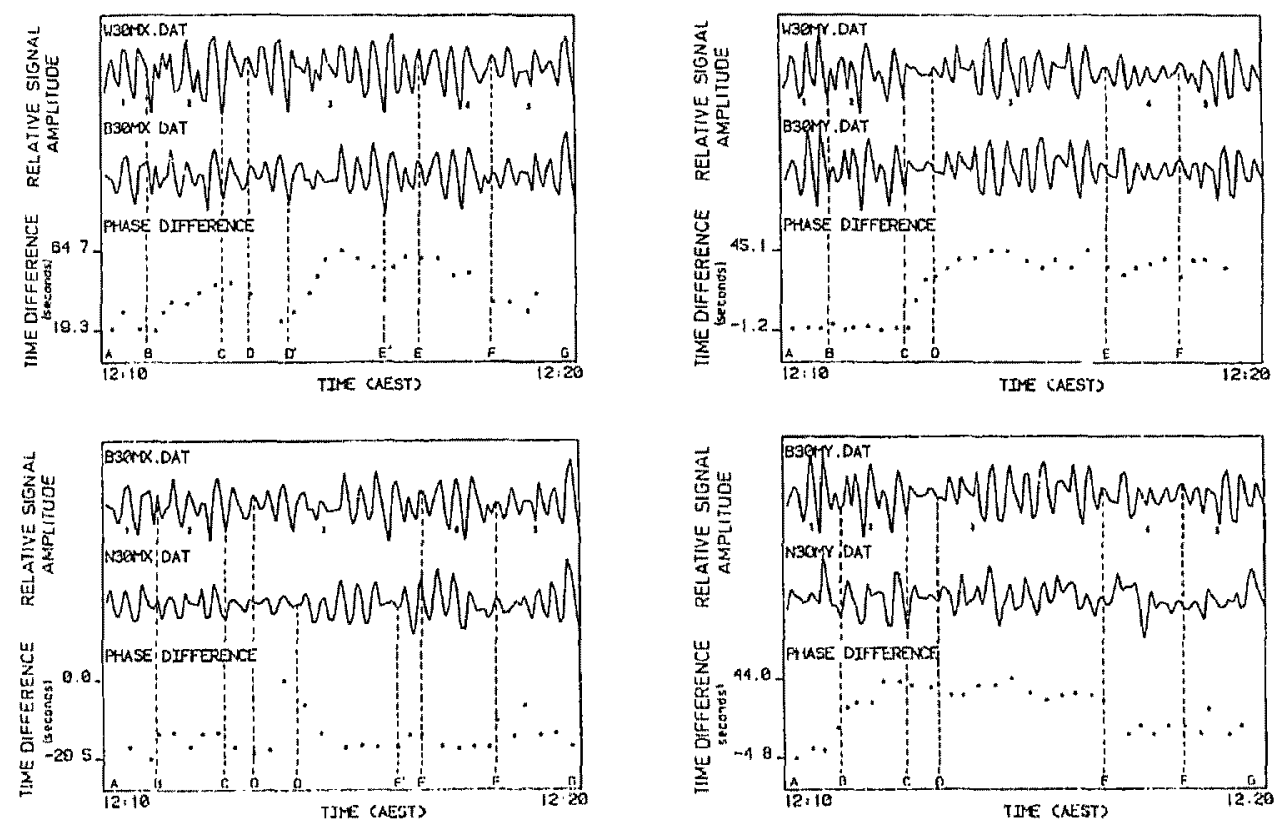

(a)

(b)

Figure 8. Interstation wave packet structure for $1210-1220 \mathrm{hr}$ AEsT on $30 \mathrm{May}, 1982$ for the WM-BH and BH-NC pairs of stations. (a) $X$ component data, (b) $Y$ component data.
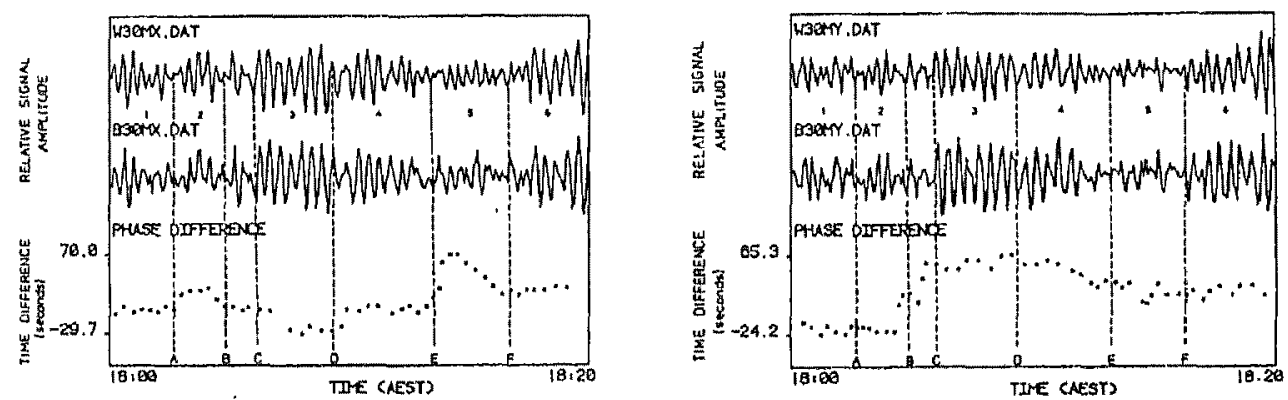

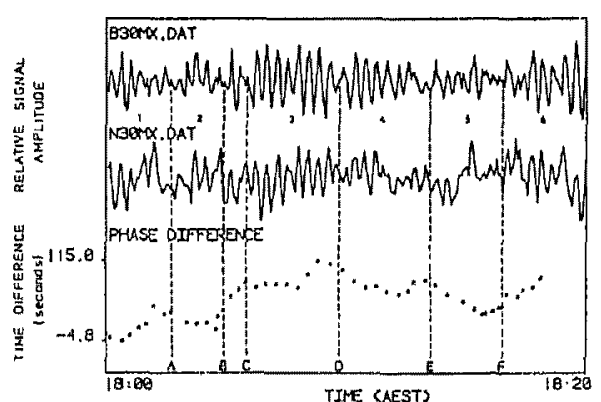

(a)

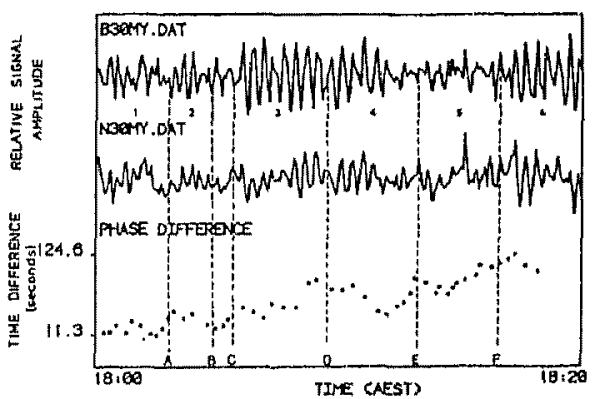

(b)

Figure 9. Interstation wave packet structure for $1800-1820 \mathrm{hr}$ AEST on $30 \mathrm{May}, 1982$ for the WM-BH and BH-NC pairs of stations. (a) $X$ component data, (b) $Y$ component data. 
geometry, the field lines at different stations could have slightly different natural frequencies and, as a consequence of this, the signals drift out of phase and they also damp out because of either collisionless or ionospheric damping (Newton et al 1978). In order to provide a new impulse for each wave packet these impulses must occur every three minutes or so. This impulsive process suggested by Mier-Jedrzejowicz and Hughes could not explain our observations as phase shifts seldom occur simultaneously at all latitudes.

A second explanation by Mier-Jedrzejowicz and Hughes suggests that there may be sharply delineated regions, each with its own field line resonance, which may differ in frequency and phase. These regions may be moving in longitude relative to the station. According to this explanation the boundary of the resonance region should move sufficiently fast so as to reach all the three longitudinal station instruments. Taking into consideration the resolution of the recording instrument and the distance between stations, the azimuthal speed comes out to be about $150 \mathrm{~km} / \mathrm{sec}$ which is a reasonable speed for an impulse, but not for the boundary of a region which then resonates for three minutes. Hence this explanation is also not surtable to explain our observations.

According to the third explanation by Mier-Jedrzejowicz and Hughes the motion of a resonance region in latitude past the station would cause a sudden change in polarization which in turn would move power among the components of a signal. A slow rotation of the polarization ellipse would produce an attenuation of the signal in one component and a growth in the other. Because of the gradual change in the relative phase of two components, it would appear that the frequency of the signal was changing when only one component was observed. A rapid change in polarization would, therefore, cause apparent phase skips.

This interpretation also cannot explain our observed phase skips because a phase skip in one component due to a sudden change of polarization must be accompanied with a corresponding skip in one or both of the other components and this does not always happen as is obvious from our results.

Lanzerotti et al (1981) also observed many sudden phase shifts when studying data from three low latitude Californian stations at $L \approx 2$. The phase shifts were found to be variable between components and stations. They also argue against the impulsive source process.

The results for the azimuthal stations at wM, BH and NC show similar properties to those found by Lanzerotti et al (1981). It was difficult to define distinct wave packets which showed the same time occurrence pattern at all three stations. It appears that corresponding signal components between different stations often show a greater similarity in wave packet structure than do the two components at a single station (figure 8). Sudden changes in phase or phase jumps are seen at all three stations in both components but like Lanzerotti's and coworkers' data no systematic pattern was observed between components or stations. The phase differences between pairs of stations were mostly variable and no consistently linear frequency drifts such as those expected as a consequence of a free resonant shell oscillation in the absence of a driving force were observed. On many occasions a constant phase was observed over individual packets but again no consistent pattern between stations and components was evident. More examples of stable phases were observed between WM and BH stations which were spaced $425 \mathrm{~km}$ apart than between the BH-NC pair spaced $1065 \mathrm{~km}$. This may indicate that phase variations seen on the ground have short scale lengths.

The ionosphere modifies the magnetospheric wave signals seen at the ground 
(Hughes 1974). It is therefore conceivable that some of the phase difference patterns seen in the experimental data may be caused by amplitude and phase variations arising from low and middle latitude ionospheric currents and irregularities. Furthermore signals observed on the ground may arise from a combination of individual $L$-shell oscillations which could also produce confusing spatial and temporal phase patterns.

\section{Acknowledgements}

This research was supported by grants from the Australian Research Grants Scheme and the University of Newcastle. One of the authors (IAA) was supported by a University of Newcastle Scholarship and is also thankful to the Department of Electrical and Computer Engineering of the University of Wollongong for assistance.

\section{Appendix}

\section{Computer programs}

Brief descriptions of the computer programs are given here.

\section{CONTPT: contour plots}

Input to the program consists of a single channel $(X$ or $Y)$ of data. The power spectrum is calculated using the FFT method. A three-point smoothing routine SMCOrH was also developed to smooth power spectral densities for better representation. There are two versions of the main program, one is employed for eight hour data blocks and the other for four hour data blocks. The subroutine CNTPLT was written to arrange the power spectral density to be stored in the form of equispaced grid points in a two dimensional field.

CONTOUR is a subroutine package available for use on the vax-11/780 computer in conjunction with IGL. The subroutine contour plots contours of the power spectral density function $P=f(f r, t)$ in a two-dimensional graph when values of $P$ are specified at grid points which are equispaced in the fr (frequency) and $t$ (time) directions. Therefore the total size of the rectangular array is determined by IDATA and JDATA, the number of grid points in the $f r$ and $t$ directions, respectively.

A procedure of interpolation is employed to plot a continuous contour line. The maximum and the minimum values of the power spectral densities are specified and four contour levels are computed. The output to be plotted is sent to an IGL Capture/Replay file CONTUR.DAT. The plots are then obtained on a Tektronix$4025 / 4612$ graphics terminal and on the plotter at the University of Newcastle computing centre.

\section{WPS: wave packet structure}

This program plots a time series $(X$ or $Y$ ) data file. It also plots the wave cycle number count on the vertical axis using the IGL plotting routines. The output to be plotted is sent to an IGL Capture/Replay file. The plots were normally obtained on the Tektronix$4025 / 4612$ graphics terminal and on the plotter at the University of Newcastle computing centre. 
PSHIFT: phase shift

The program plots two time series $(X$ and $Y$ ) data files. It also plots the time difference between the corresponding signal peaks on the vertical axis by employing the IGL plotting routines.

\section{References}

Ansari I A 1984 Multistation observations of low latilude Pc3 geomagnetic pulsations Ph.D thesis, University of Newcastle

Herron T J 1966 J. Geophys. Res. 71871

Hughes W J 1974 Planet. Space Sci. 221157

Jacobs J A 1970 Physics and chemistry in space (Berlin: Springer-Verlag) Vol I, p. 176

Lanzerotti L J, Medford L V, MacLennan C G and Hasegawa T 1981 J. Geophys. Res. 865500

Mier-Jedrzejowicz W A C and Hughes W J 1980 J. Geophys. Res. 856888

Newton R S, Southwood W J and Hughes W J 1978 Planet. Space Sci. 26201

Olson J V and Rostoker G 1978 J. Geophys. Res. 832841

Southwood D J 1975 Geophys. J. R. Astron. Soc. 41425 